\title{
Labyrinthe
}

$36 \mid 2011$ (1)

«Par les Grecs »

\section{Quelques pas à travers de longues routes sinueuses}

\section{Pietro Pucci}

Traducteur : Traduit de l'italien par Renaud Pasquier

\section{(2) OpenEdition}

Journals

Édition électronique

URL : http://journals.openedition.org/labyrinthe/4117

DOI : $10.4000 /$ labyrinthe.4117

ISSN : 1950-6031

Éditeur

Hermann

Édition imprimée

Date de publication : 15 février 2011

Pagination : 33-36

ISBN : 9782705680541

Référence électronique

Pietro Pucci, «Quelques pas à travers de longues routes sinueuses », Labyrinthe [En ligne], 36 | 2011

(1), mis en ligne le 01 février 2013, consulté le 05 mai 2019. URL : http://journals.openedition.org/

labyrinthe/4117; DOI : 10.4000/labyrinthe.4117

Propriété intellectuelle 


\section{Quelques pas \\ à travers de longues routes sinueuses}

Pietro PUCCI

Contact:pp26@cornell.edu

Suivons un instant le chemin d'EEdipe dans l'EEdipe-Roi de Sophocle, suivons-le en accompagnateurs contemporains du roi - il faudrait plus exactement l'appeler tyran - et donc en lecteurs historiens et philologues. Puisque trois mille ans environ nous en séparent, la reconstruction de ce chemin n'est rendue possible (?) que par l'immense réserve d'informations, de textes, de méthodes qui s'est accumulée pendant tout ce temps: par une énorme bibliothèque, en somme. Trois mille ans de livres voilent et dévoilent les empreintes de ces pas. Et nous, nous sommes en chemin avec eux, sur elles, ajoutant livres et traces. Dépassons l'ambition historique et scientifique, considérable, dépassons le projet évidemment utopique; ne nous arrêtons même pas pour suspecter la narration historique, parce que, on le sait, les faits décisifs ne peuvent être reconnus comme tels qu'une fois découverts tous les faits, le telos, c'est-à-dire le fil conducteur des faits historiques tiré d'une représentation ou d'une métaphysique qui gît en dehors de ces faits, etc. Pourtant chacun, tous, nous nous racontons notre histoire, sans arrêt. Tous, nous refaisons le chemin en arrière, nous repartons de notre et père et de notre mère, comme le fit Edipe, pour savoir - ou en croyant pouvoir savoir - qui nous sommes.

Alors suivons Edipe, notre modèle, compagnon de voyage, comme si le texte avait été écrit hier. Laissons là le scandale et avançons juste d'un pas ou deux dans cette direction. La reconstruction la plus patiente et la plus rigoureuse ne comblera pas les vides de la représentation artistique, son rapport abyssalement oblique et distrait avec la réalité. Et puis le langage poétique possède sa propre continuité, sa propre permanence. Pour que telle assertion ne paraisse pas naïve, référons-nous à la tradition, et citons le vers d'Horace: 


\section{Quid obliquo laborat lympha fugax trepidare rivo ?}

L'interprétation la plus rigoureuse que l'on puisse fournir à propos de faits historiques, à propos, mettons, de l'épicurisme horatien et de son éclectisme, ne rendra jamais compte d'une image aussi immédiate et palpitante: un tel effet dépend en partie d'éléments formels, comme entre autres le fait, purement accidentel, qu'un mot comme trepidare ait perduré dans les langues romanes, et perduré comme mot poétique. Ainsi, cette lymphe fuyante qui s'épuise à courir, toute trépidante, dans le tortueux ruisseau, appartient à un langage poétique traditionnel avec ses métaphores, allitérations, doubles sens, etc., qui vit depuis des millénaires, et traverse obliquement les parcours et discours historiques, toujours indemne, ou presque.

Réglons nos pas sur ceux d'CEdipe. Depuis que Freud en a fait l'exemple du complexe œdipien, il est devenu le juge-tyran de tout discours critique, littéraire ou pas, et de tout parcours entre nature et culture. Il n'est plus possible, pour le chercheur antiquisant, d'ignorer ce phénomène, et pas seulement par peur de s'exclure d'un dialogue avec le monde moderne, mais surtout parce que le thème freudien est bien là, déjà, dans la nécessaire transgression accomplie par le fils. Dans la mesure où la transgression est prédite par Apollon, elle est posée comme inévitable, et comporte donc non seulement l'interdiction, mais avant tout la suppression de tout ce qui est individuel, causal, accidentel, innocent, non voulu, dans l'histoire réelle d'Eedipe : de fait, dans l'acte de la transgression œdipienne, l'interdiction apparait alors qu'elle est brisée, et la faute s'ensuit. On ne pourra nier qu'Edipe parait à la fois fasciné et dégoûté par l'oracle d'Apollon: tant d'acharnement et tant de violence pour fuir ce vers quoi il va à coup sûr, par des voies détournées et fortuites.

Dire que le thème freudien est là, y compris le rêve incestueux des enfants, ne veut pas encore dire accepter l'interprétation que Freud en donne dans L'Interprétation des rêves... Des problèmes de tous ordres se présentent, difficultés spécifiques au thème, comme par exemple la notion freudienne de « complexe », fort problématique, ou les deux interprétations différentes que Freud donne de l'aveuglement d'CEdipe et les variations sur les thèmes œdipiens que les psychanalystes - hommes et

1. «Pourquoi l'ombre furtive bondit-elle avec effort dans le lit sinueux du ruisseau? », Horace, Odes, II, 3, trad. F. Lasserre, Paris, Belles Lettres, 1967. 
femmes - ne cessent d'élaborer, cédant souvent à des tentations opposées : tantôt ils interprètent les moments du drame avec pour preuve le dossier de leurs cas cliniques, tantôt ils schématisent les moments, les figures, les stratégies du drame en larges symboles - ce qui fit dire, récemment, à Nicole Loraux, que la psychanalyse était chose trop importante pour être laissée aux mains des psychanalystes. Demeurent donc de graves difficultés d'ordre méthodologique. La première concerne les notions de complexe, inconscient, répression, sexe, etc., qui n'ont pas de traductions grecques: les équivalents grecs ont leurs propres contextes et spécificité. « Sexe » en grec se dira Éros ou Aphrodite, ce qui donne la mesure de la différence. La seconde, liée à la première, touche au langage grec, à la spécificité duquel il faudrait rattacher toute interprétation, surtout la psychanalytique. J'entends par " langage » plus particulièrement le langage du drame en question: analyser les composantes rhétoriques et textuelles qui sous-tendent ses images, les déterminent, et que bien souvent le psychanalyste ne définit que par larges symboles universels. Un exemple suffira: dans le texte de l'CEdipe-Roi la production et la génération des fils sont souvent pensées comme événements dus au hasard (tukhè) - qui ne se souvient d'CEdipe, fils de la Tukhè -; mais la production de la parole est elle-même représentée par la métaphore de la génération - et donc du hasard - non sans conséquences remarquables sur le thème œdipien. On ne s'étonnera pas que, selon Tirésias, ce soit précisément le hasard, ou plutôt un événement du hasard, qui a permis à Edipe de résoudre l'énigme, et, en toute cohérence, que, selon le chœur, les lois sacrées ne soient pas engendrées par la mortelle nature, mais seulement par le Père Olympien. Mais alors, ce nœud métaphorique entre la parole (celle, véridique, d'Apollon, passe aussi à travers des bouches humaines) et la génération, fortuite, par des semences humaines devrait paraître problématique, suspect. À quelle nature appartient la parole de Tirésias? À travers quelles routes acquiert-elle force et valeur? Il ne serait pas impossible de lire une certaine « dissémination » dans ce nœud métaphorique et de considérer le message d'Apollon - la nécessité de la transgression - comme un après coup pour contrôler la dissémination linguistique, celle des générations, etc.

Et revoici la question avec laquelle nous avions commencé, celle de la nature du langage poétique, et de la façon dont le discours critique en répète la force permanente, qui est aussi, paradoxalement, force de dérive, comment donc celui-ci mime le littéraire, évoluant à travers de 


\section{Labyrinthe, $n^{\circ} 36$}

longues routes sinueuses sur les traces de l'autre, dispensant les pensées du texte, tandis qu'il se dissémine continuellement.

On ne peut ainsi affirmer clairement que l'CEdipe-Roi montre l'échec du rationalisme grec du cinquième siècle, ou qu'il témoigne de son pouvoir, ou qu'il mette à l'épreuve ses ressources: dans l'esquisse que j'en ai fournie, le pouvoir de la parole fortuite, disséminante, distordante articule également, pour le lecteur, l'oracle d'Apollon et en dénature la fonction et le but. L'oracle a dit la vérité sur CEdipe, contre Edipe : mais cette vérité rétroactive n'a rien fait d'autre que de marcher sur les articulations du langage du hasard, de la tukhè. Il faudra au moins reconnaître à Sophocle ou à son texte une notion perverse du langage de la vérité. Mais l'on peut encore aller plus loin...

On peut encore continuer, à travers de longues routes sinueuses...

Traduit de l'italien par Renaud Pasquier 